Relations industrielles

Industrial Relations

\title{
DeBresson, Christian, Comprendre le changement technique
}

\section{Martin Dumas}

Volume 49, numéro 1, 1994

URI : https://id.erudit.org/iderudit/050930ar

DOI : https://doi.org/10.7202/050930ar

Aller au sommaire du numéro

\section{Éditeur(s)}

Département des relations industrielles de l'Université Laval

\section{ISSN}

0034-379X (imprimé)

1703-8138 (numérique)

Découvrir la revue

Citer ce compte rendu

Dumas, M. (1994). Compte rendu de [DeBresson, Christian, Comprendre le changement technique]. Relations industrielles / Industrial Relations, 49(1), 202-203. https://doi.org/10.7202/050930ar

Tous droits réservés (C) Département des relations industrielles de l'Université Laval, 1994
Ce document est protégé par la loi sur le droit d'auteur. L’utilisation des services d'Érudit (y compris la reproduction) est assujettie à sa politique d'utilisation que vous pouvez consulter en ligne.

https://apropos.erudit.org/fr/usagers/politique-dutilisation/ 
praise for their active support of and involvement in the implementation of EP's, employers' behavior is severely criticized as being obstructive (p. 60) and short-sighted (p. 68): employers' "willingness to top up redundancy payments in order to buy off those employees interested in the 'new' ideas, seem to be greater than their courage in implementing new personnel policies. This conservatism reflects their ideological inability to manage the implementation of employment plans, either on the organisational or conceptual level. Only a handful of personnel managers in the Federal Republic of Germany seem to possess the skills that this task would require" (p. 166). Instead of such generalizations, the reader would have preferred a more careful analysis of the positions, perspectives, and interests of all the actors involved in the establishment and implementation of $E P$ 's. For the further development of $E P$ policies such a more careful analysis seems to be all the more important since the power of unions and works councils to exert pressure on management to participate in $E P$ policies may be substantially weakened and, therefore, more consensual arrangements may be more promising when EP's are needed most, - in times of economic crisis.

Christoph F. Buechtemann

RAND, Santa Monica, California

Comprendre le changement technique, Christian DeBresson, Ottawa, Les Presses de l'Université d'Ottawa, Éditions de l'Université de Bruxelles, 1993, 386 p., ISBN 2-7603-0336-5 (PUO), ISBN 2-8004-1069-8 (ÉUB).

Les écrits scientifiques qui, d'abord surspécialisés, doivent à leur seule nouveauté d'être reçus par la communauté savante n'ont pas grand peine à être reçus, mais en ont à être gardés en mémoire. Tel ne sera certes pas le sort de l'ouvrage soumis à ma lecture. Ses qualités, incomparables à celles d'un expédient devant l'absence de manuel introductif en matière de changement technique, servent la remise en question de la catégorisation contemporaine du savoir en ce domaine. Avec la finesse d'un esprit nuancé et la puissance d'une solide érudition, DeBresson arrive en effet à offrir du changement technique une vision entière qui défie toute frontière disciplinaire. J'examinerai successivement la trame, les assises ainsi que la portée de Comprendre le changement technique.

Bien que l'auteur se défende de favoriser une approche particulière à l'étude de la technologie aux dépens d'autres, son discours s'articule autour de l'objectif déclaré de « démocratriser le choix technique ». A posteriori, il n'y a pas là de contradiction dans la mesure où la légitimité d'une telle entreprise repose sur une étude approfondie des faits pertinents envisagés sous divers angles. Ainsi, le lecteur est amené à suivre une pensée qui intègre, dans un langage sobre et clair, les facteurs psychologiques, sociaux, économiques et politiques qui interagissent avec le choix, le développement et la diffusion des techniques. Il ressort avant tout du propos que le changement technique, loin de tracer inéluctablement la voie neutre du progrès à la manière du fatalisme, procède de choix réalisés par un nombre restreint d'individus (fonction du degré de concentration du pouvoir politique et économique) et orientés par de multiples contraintes et incitatifs (e.g. processus d'apprentissage, valeurs culturelles, demande, réduction des coûts, 
recherche de prestige et de pouvoir, etc.). La démocratisation du choix technique est d'abord démystification systématique.

Les assises théoriques de l'ouvrage sont plurielles et, le plus souvent, classiques. Inspiré par des penseurs tels que Smith, de Tarde, Bloch, Veblen et Usher, DeBresson retrace les grands débats qui, de près ou de loin, ont influencé notre compréhension du changement technique. L'effort valable de théorisation, bien que forcément limité, ne présente pas le défaut de l'irréalisme. De nombreuses illustrations empiriques, et plus particulièrement des études de cas, étaient le propos de l'auteur. Entre autres exemples, mentionnons les expériences de Rutherford (particularités du développement technique), Bombardier (processus d'élargissement de la demande), Ford (mise à profit du savoir-faire ouvrier) ainsi que l'historique de la bielle-manivelle (liens unissant systèmes techniques et organisations sociales). Aussi le lecteur est-il invité à passer à l'action en répondant aux questions-synthèses exposées à la fin de chacun des vingt chapitres.

Aux yeux d'un intervenant en relations industrielles, la portée de l'ouvrage peut sembler déborder, à prime abord, le champ de ses préoccupations quotidiennes. Je retiens trois domaines d'intérêt qui se situent à la croisée de ce que DeBresson offre et de ce qui pourrait capter l'attention des managers, syndicalistes, décideurs politiques et théoriciens du social. Le premier renvoie à l'organisation du travail, voisine de la technique entendue comme une "façon de faire ». Une lecture attentive de l'ouvrage conduira à reconnaître l'impact des relations sociales structurées et vécues sur les lieux de travail sur le sens du changement technique, ce dernier découlant d'un « consensus social, implicite ou explicite ». Le deuxième rejoint l'objectif de démocratisation du choix technique dans un contexte économique difficile. L'ouvrage, en plus de tirer les grands traits de l'effet sur l'emploi des ordinateurs et des semi-conducteurs - dissociés sensément de la «nouvelle technologie » - offre une vision optimiste de l'avenir en faisant la démonstration que les choix sociaux, cristallisés en partie par les voies institutionnelles, sont maîtres du sens donné au progrès. A cet égard, et considérant l'importance accordée à l'augmentation de la productivité par le biais de l'introduction de nouveaux équipements et de nouvelles qualifications, l'ouvrage de DeBresson constitue un cadre utile à la réflexion. Le troisième domaine d'intérêt renvoie plus largement à l'enrichissement culturel personnel; le lecteur curieux trouvera matière à satisfaction.

En somme, Comprendre le changement technique constitue un appel à la responsabilisation fondé sur l'hypothèse, solidement enracinée, que le sens du progrès est à notre portée. Sa lecture suffit à convaincre que le deus ex machina de nos problèmes socio-économiques n'est bel et bien qu'une création médiatique.

Martin Dumas 